\title{
Intrabulbar Projecting External Tufted Cells Mediate a Timing-Based Mechanism That Dynamically Gates Olfactory Bulb Output
}

\author{
Zhishang Zhou and Leonardo Belluscio \\ Developmental Neural Plasticity Unit, National Institute of Neurological Disorders and Stroke-National Institutes of Health, Bethesda, Maryland 20892
}

In the mammalian olfactory system, intrabulbar projections (IBPs) mediated by a class of external tufted cells (ET cells) specifically link isofunctional odor columns within the same olfactory bulb. To study the function of these ET cells within the glomerular network, we developed a "hemibulb" preparation that maintains IBPs intact enabling the select activation of ET cells associated with specific glomeruli. Using P2-GFP mice, a line in which the P2 glomeruli are labeled with green fluorescent protein, we recorded from P2 mitral cells (MT cells) while selectively stimulating P2 ET cells. Here, we show that ET-cell activity evokes a slow modulatory (SM) potential within MT cells, which is mediated by the glomerular network and consists of both excitatory and inhibitory components. Interestingly, the timing of the SM potential with respect to olfactory nerve $(\mathrm{ON})$ stimulation can produce converse effects on MT-cell output. When ET-cell activity precedes ON stimulation, the MT-cell response is potentiated; however, when ET-cell activity follows ON stimulation, the MT-cell response is inhibited. Thus, intrabulbar projecting ET cells can shape olfactory bulb output through intraglomerular modulation of MT cells.

Key words: olfactory bulb; intrabulbar; glomerulus; external tufted cells; chemosensory; mice

\section{Introduction}

Olfactory sensory neurons (OSNs) detect odorant stimuli from the outside world and relay the information directly to the main olfactory bulb, which in turn sends projections to the olfactory cortex. Within the bulb, the information is processed by a diverse network of interneurons that regulate the transfer of sensory input as it flows from the periphery to higher brain regions. Although the precise nature and exact location of these regulatory sites is widely unknown, it is generally believed that the glomerulus, as a local communication hub for most cell types within the bulb, plays a central role in the regulatory process (Chen and Shepherd, 2005).

Studies have shown that each bulb contains two mirrorsymmetric glomerular maps, in which the functional identity of each glomerulus is determined by the odorant receptor expressed by the OSNs projecting to it (Ressler et al., 1994; Vassar et al., 1994; Mombaerts et al., 1996). A second-order map also exists in which external tufted cells (ET cells) connect the glomerular maps through a set of reciprocal projections that specifically connect isofunctional odor columns (Belluscio et al., 2002; Lodovichi et al., 2003). Thus, we explored the possibility that ET cells are

Received July 2, 2008; revised August 5, 2008; accepted August 28, 2008.

This work was supported by the Intramural Research Program of the National Institute of Neurological Disorders and Stroke-National Institutes of Health. We thank Beth Belluscio, Rory McQuiston, and John Isaac as well as the members of the Belluscio laboratory for helpful discussions and critical review of this manuscript.

Correspondence should be addressed to Leonardo Belluscio at the above address. E-mail: belluscl@ninds.nih.gov.

DOI:10.1523/JNEUROSCI.3082-08.2008

Copyright $\odot 2008$ Society for Neuroscience $\quad$ 0270-6474/08/289920-09\$15.00/0 involved in regulating olfactory information flow by modulating glomerular output.

The glomerular network was initially considered to play an inhibitory role in the transfer of sensory information primarily attributable to the numerous inhibitory neurons surrounding each glomerulus (Shepherd et al., 2004). However, several studies have also identified many cells in or near the glomerular region, including ET cells, that are excitatory neurons resulting in a more complex view of glomerular function (Shepherd, 1963; Freeman, 1974; Wellis and Scott, 1990). Recent studies probing the electrical properties of ET cells have reported that they have an intrinsic rhythmic-bursting ability (McQuiston and Katz, 2001; Hayar et al., 2004a; Antal et al., 2006; Zhou et al., 2006) and that ET cells associated with a given glomerulus are able to synchronize their activity through various intraglomerular mechanisms that are not thoroughly understood (Hayar et al., 2004b, 2005). We suggest that this coordination serves to regulate the flow of olfactory information through the glomerulus. ET cells receive direct olfactory nerve (ON) input and synapse directly with periglomerular cells (PG cell). Importantly, OSNs, and PG cells also synapse onto mitral cells (MT cells), the main projection neurons of the bulb. Thus, we hypothesized that ET cells regulate olfactory bulb output by modulating MT-cell activity through the glomerular network.

Here, we have developed a preparation enabling us to study the influence of intrabulbar projecting ET cells on olfactory bulb output. Our in vitro slice preparation preserves the intrabulbar link between isofunctional glomeruli which makes it possible to selectively activate the ET cells associated with a specific target 

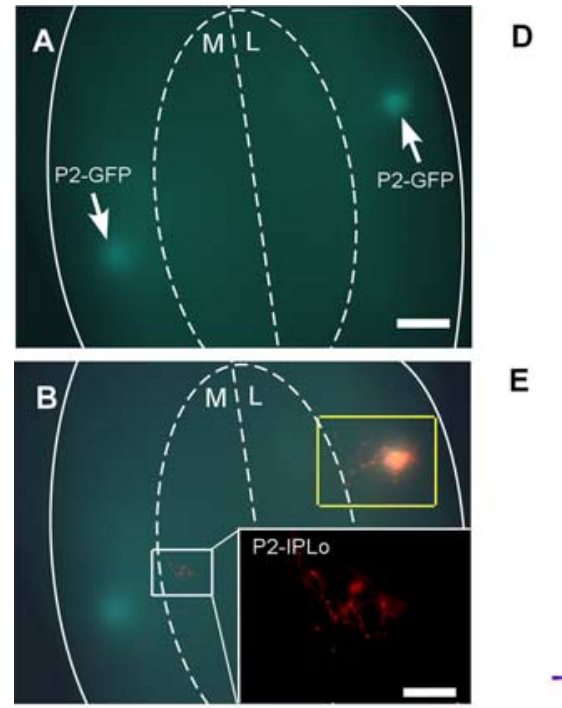

E
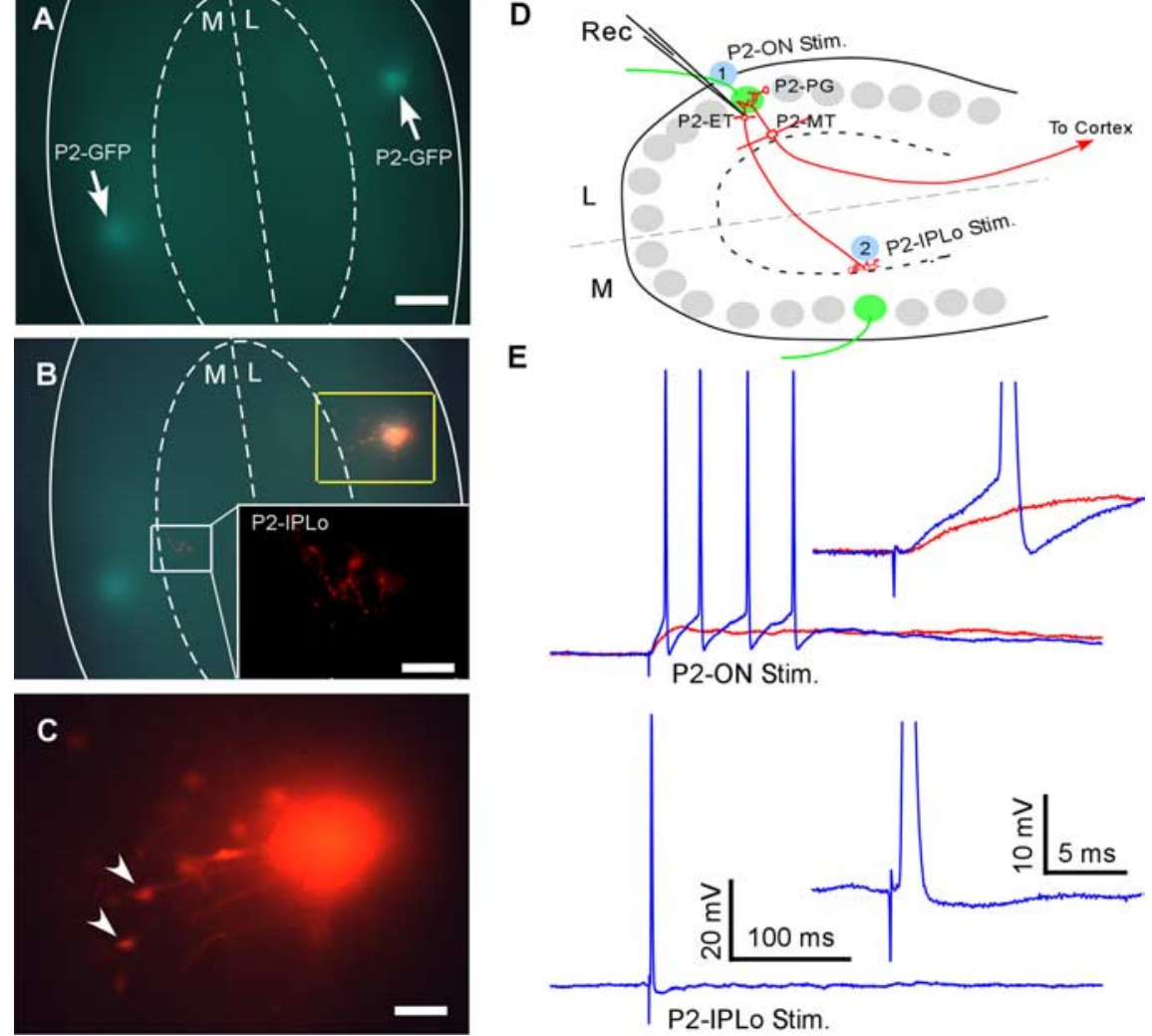

Figure 1. Hemibulb preparation enables the targeted excitation of ET cells associated with specific glomeruli through IPLo stimulation. $\boldsymbol{A}-\boldsymbol{C}$, HB slice preparation from a P2-GFP mouse. $A$, Fluorescent image of HB slice containing two isofunctional P2 glomeruli (arrows). The solid white lines indicate the outer boundary of the HB slice. The dotted curved line indicates the estimated border of mitral cell layer and internal plexiform layer. The dotted straight line represents the axis of symmetry between the medial and lateral halves of the bulb. $\boldsymbol{B}, \mathrm{HB}$ slice after rhodamine dextran injection targeting the lateral P2 glomerulus (yellow boxed region and $\boldsymbol{C}$ ). The labeled axon terminals of the P2 intrabulbar projections (white boxed region) form the P2-IPLo site beneath the medial P2 glomerulus (inset). C, Close-up of the rhodamine injection site from $\boldsymbol{B}$ showing labeled P2-mitral cells (arrowheads). D, Schematic of experimental configuration to confirm that P2 intrabulbar projections were functionally intact. Gray dashed line, Axis of symmetry between the medial and lateral halves of the bulb. Black dashed line, The boundary between that MT layer and the IPL. Whole-cell recordings were taken at P2-ET-cell bodies, whereas electrical stimulation was delivered to either the outer nerve leading to the P2 glomerulus (blue dot 1 ) or the P2-IPLo (blue dot 2). E, Whole-cell recording from a P2-ET cell (resting membrane potential, $-56 \mathrm{mV}$ ). Top traces show transsynaptic activation of a P2-ET cell by stimulating the P2-ON using $50 \mu \mathrm{A}, 0.2 \mathrm{~ms}$ (blue trace) and $30 \mu \mathrm{A}, 0.2 \mathrm{~ms}$ (red trace). Each stimulus elicits a delayed EPSP; the $50 \mu$ A stimulation evokes action potentials. Inset, Traces show expansion of the first evoked potentials. Bottom trace, Antidromic activation of the same P2-ET cell through P2-IPLo stimulation using a $0.22 \mathrm{~mA}$ for $0.2 \mathrm{~ms}$ resulting in an evoked action potential. Inset trace, Expansion of the action potential. This confirms that the intrabulbar projections are maintained functionally intact in this $\mathrm{HB}$ preparation. Scale bars: $\boldsymbol{A}, 200 \mu \mathrm{m} ; \boldsymbol{B}$ (inset), $50 \mu \mathrm{m} ; \boldsymbol{C}, 50 \mu \mathrm{m}$. M, Medial; L, lateral; Stim, stimulation.

glomerulus through antidromic stimulation. Using P2-GFP mice, we determined that the activation of the intrabulbar projecting ET cells is capable of eliciting both excitation and inhibition of MT cells through the intraglomerular network. Interestingly, we find that timing of this activation with respect to primary sensory input plays a critical role in determining whether MT-cell output is potentiated or suppressed.

\section{Materials and Methods}

Slice preparation. All experiments were performed on P2-GFP mice, a transgenic line in which GFP is coexpressed in all P2 odorant receptor neurons (Feinstein and Mombaerts, 2004). Olfactory bulbs were acutely removed from 4-to 9-week-old mice as described previously (Zhou et al., 2006), and hemibulb (HB) slices were prepared such that $P 2$ intrabulbar projections were maintained intact. Briefly, mice were first anesthetized with ketamine $(100 \mathrm{mg} / \mathrm{kg})$ and then intracardially perfused with $4^{\circ} \mathrm{C}$ modified artificial CSF (ACSF) ( $\mathrm{NaCl}$ was replaced by equal osmolar sucrose). Olfactory bulbs were dissected in ACSF containing (in $\mathrm{mM}$ ):
$124 \mathrm{NaCl}, 3 \mathrm{KCl}, 1.3 \mathrm{MgSO}_{4}, 2 \mathrm{CaCl}_{2}, 26$ $\mathrm{NaHCO}_{3}, 1.25 \mathrm{NaH}_{2} \mathrm{PO}_{4}, 10$ dextrose, and oxygenated with $95 \% \mathrm{O}_{2}, 5 \% \mathrm{CO}_{2}$. The bulbs were then positioned ventral side up on an agarose block and sectioned horizontally (Vibratome 3000 Sectioning System), producing two 700-900 $\mu \mathrm{m}$ HB slices, each resembling a half-moon and containing a pair of isofunctional $\mathrm{P} 2$ glomeruli on opposing edges with intact intrabulbar connections wrapping around the bulb between them. HB slices were incubated in a $37^{\circ} \mathrm{C}$ chamber for $\sim 30 \mathrm{~min}$ and then placed under a fluorescence microscope (BX51WI; Olympus) to identify the P2-GFP glomeruli (Fig. $1 A$ ). To visualize the precise location of the $\mathrm{P} 2$ intrabulbar projection tuft, we injected rhodamine dextran dye (Invitrogen) into either medial or lateral $\mathrm{P} 2$ glomerulus using 50-100 current pulses ( $20 \mathrm{nA}, 0.2 \mathrm{~s}, 0.5 \mathrm{~Hz})$ for 100-200 s delivered by an A360 stimulator (WPI). The slices were maintained in ACSF for $4-8 \mathrm{~h}$ at room temperature.

Electrophysiological experiments. Whole-cell recordings were performed on MT cells using an AxoClamp-2B amplifier (Molecular Devices). A temperature controller, TC-344B (Warner Instruments), was used to maintain the slice at $35^{\circ} \mathrm{C}$. Recording pipettes were fabricated with a P2000 puller (Sutter Instruments) with a 2-6 $\mathrm{M} \Omega$ resistance. The intracellular solution contained (in $\mathrm{mM}$ ): 130 potassium gluconate, 4 Mg-ATP, 0.3 Na-GTP, 0.2 EGTA, 20 HEPES. Rhodamine-labeled MT cells were targeted for recording under a fluorescence microscope. Data were acquired with Clampex 9.2 through a Digidata 1322A A/D converter (Molecular Devices). All cells with resting potentials above $-50 \mathrm{mV}$ or series resistance change $>20 \%$ during recording were discarded.

Stimulation and drug delivery. For most effective antidromic excitation of ET cells, a stimulating electrode was placed at their axonal terminals located in the internal plexiform layer on the opposite side of the bulb (IPLo) as determined by the location of the isofunctional P2 glomerulus and rhodamine-labeled ET axon terminals. Two to four hours after rhodamine dextran injection into a P2 glomerulus, axon terminals could be observed in the IPLo, facilitating the placement of the stimulating electrode (Fig. $1 B, D$ ). The stimulating electrodes were fabricated by threading two individual tungsten electrodes (FHC) with a resistance of $50-100 \mathrm{k} \Omega$ through a single glass recording electrode such that the tips emerged from the same hole and were staggered by $\sim 50$ $\mu \mathrm{m}$. Bipolar electrical stimulation was delivered by an A360 stimulator (WPI). Neurotransmitter receptor antagonists 6-cyano-7nitroquinoxaline-2,3-dione (CNQX) (20 $\mu \mathrm{M}$; Sigma-Aldrich), DL-2amino-5- phosphonovaleric acid (APV) (100 $\mu \mathrm{M}$; Sigma-Aldrich), or gabazine (100 $\mu \mathrm{M}$; Tocris Bioscience) were either uniformly delivered to the entire HB slice via bath perfusion or locally applied using pressure injection through glass pipettes (10 psi) in small volume at high concentrations (CNQX, $2 \mathrm{~mm}$; APV, $10 \mathrm{~mm}$ ). Two-barrel puff pipettes were constructed by gluing two individual pipettes pulled on a PP-830 puller (Narishige) and breaking their tips to a final size of 3-6 $\mu \mathrm{m}$ under a microscope. To deliver antagonists specifically to the glomerular circuitry, the glass pipettes were inserted into a P2-GFP glomerulus before pressure injection. A small volume of solution was locally injected inside the P2-glomerulus with the periglomerular cell bodies and glia limiting its spread. To compensate for the dilution of such a small volume of 


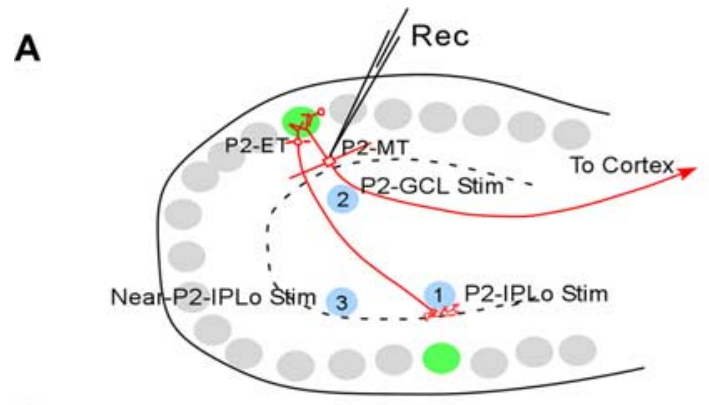

B

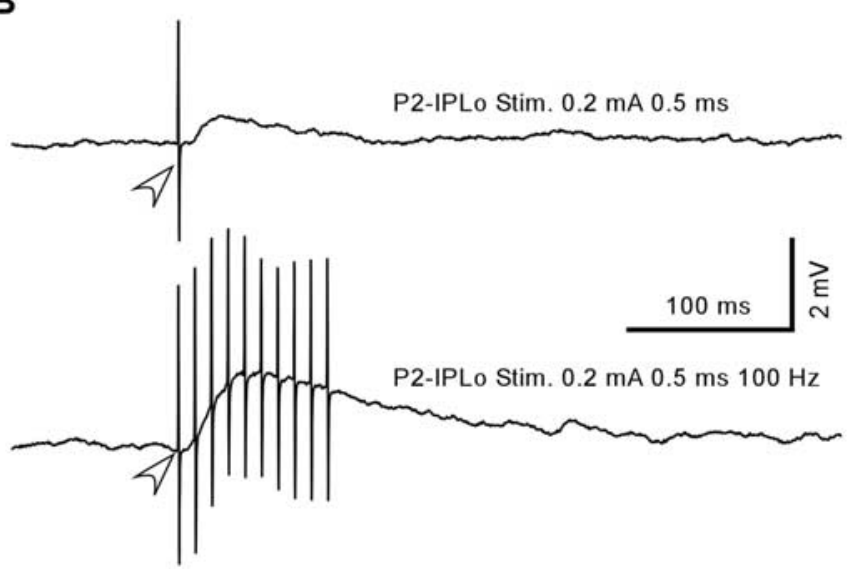

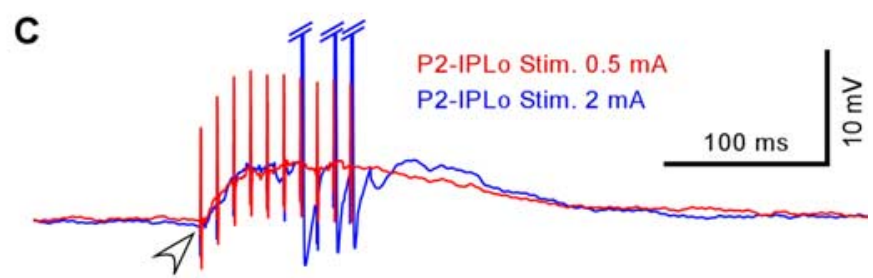

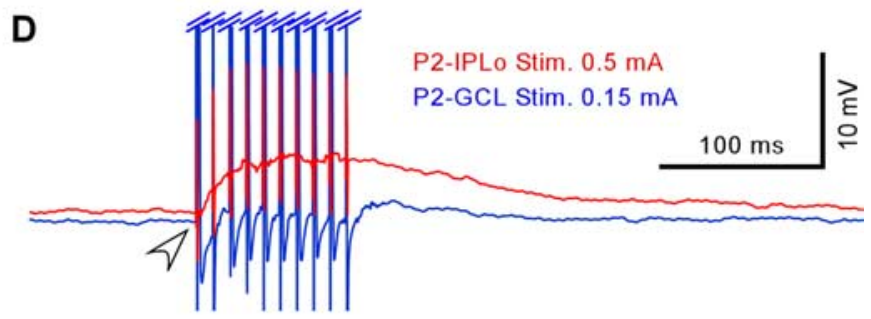

$\mathbf{E}$

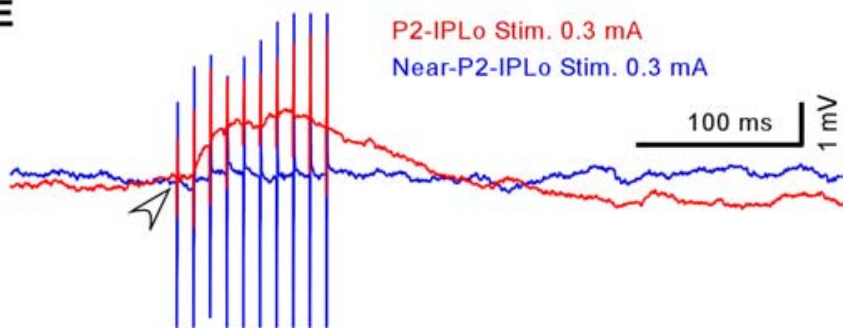

Figure 2. IPLo stimulation of P2-ET cells elicits an SM potential in P2 mitral cells. A, Diagram showing experimental configuration using HB preparation: whole-cell recordings made from P2-MT cells while stimulating either (1) P2-IPLo, (2) granule cell layer beneath the P2 glomerulus (P2-GCL), or (3) nonspecific IPLo region ( $2300 \mu \mathrm{m}$ from P2-IPLo). B, Top trace, EPSP elicited by a single P2-IPLo electrical pulse $(0.2 \mathrm{~mA}, 0.5 \mathrm{~ms})$ in the P2-MT cell with a delay of $7.4 \mathrm{~ms}$. Bottom trace, The same MT cell responding with multiple EPSPs to a train of 10 pulses at $100 \mathrm{~Hz}$, which summate to form an SM potential. Resting membrane potential (RMP), $-54 \mathrm{mV}$. C, Traces comparing moderate P2-IPLo stimulation (0.5 mA, red trace), which induced an SM potential in the P2-MT cell to a strong stimulation (2 $\mathrm{mA}$, blue trace), which elicited direct firing of the P2-MT cell seen superimposed on its SM response (diagonal lines depict truncation of action potential spikes with same designation also used in all subsequent figures). The stimulating electrode was placed in other regions of the bulb to test the specificity of P2-IPLo stimulation for inducing an SM potential. $D$, Stimulation of P2-GCL $(0.15 \mathrm{~mA}, 0.5 \mathrm{~ms}$ at $100 \mathrm{~Hz}$; blue trace) induced large action potentials in P2-MT cells but no obvious SM potential compared with P2-IPLo stimulation (red trace). E, Stimulation of an IPLo region neighboring the P2-IPLo site also failed to elicit an SM potential in P2-MT cells (blue trace) compared with P2-IPLo stimulation (red trace), confirming the specificity of the IPLo-induced SM potential. RMPs: $\boldsymbol{C}, \boldsymbol{D}, \boldsymbol{E},-51 \mathrm{mV}$. Stim, Stimulation.

neurotransmitter blockers while still maintaining an effective receptor antagonist dose, we used a 100-fold higher concentration in the pipette than we used in the bath perfusion. These injection parameters were determined using rhodamine dextran dye, which was included in the puff pipette enabling us to visualize the spatial extent of the injection, and to determine the selectivity and effectiveness of the blockers. Data collection, stimulation, and drug delivery were controlled via a Master- 8 timer (AMPI).

Data analysis. All electrophysiological data were initially analyzed with Clampfit 9.2 (Molecular Devices). Curve fitting, spike sorting, and other analysis was done with Igor Pro 5.0 (Wavemetrics). Statistical analyses were performed with Microsoft Excel and comparison done using Student's $t$ test with $p<0.05$ considered significant. All group data were presented as mean $\pm \mathrm{SD}$. All $n$ values represent cell number unless otherwise specified.

\section{Results}

\section{Hemibulb preparation enables the selective activation of ET} cells associated with specific glomeruli

Efforts to tease apart the role of specific cell types within the glomerular structure have been complicated in part because of the large variety of neurons within the periglomerular region. Distinguishing one neuronal cell type from another is often difficult, and activating multiple cells of one type is even more challenging. To address this issue, and to gain a better understanding of the role that intrabulbar projection neurons play within the glomerular circuitry, we developed an in vitro slice preparation that allows us to selectively activate groups of ET cells associated with a given target glomerulus. Studies have shown that, unlike mitral and middle tufted cells which send their axons out of the olfactory bulb toward the cortex, ET cells project their axons to the IPLo such that they specifically link isofunctional glomeruli through their associated odor column (circuitry that lies beneath each glomerulus) (Willhite et al., 2006). Thus, we reasoned that by delivering a small local stimulus to the IPL beneath a given glomerulus, the antidromic signal would selectively activate the ET cells associated with its partner glomerulus on the opposite side of the bulb through antidromic activation.

Utilizing P2-GFP mice, we prepared a hemibulb slice in which the isofunctional pair of $\mathrm{P} 2$ glomeruli can be visualized using a fluorescence microscope (Fig. 1A). We then injected dextranrhodamine tracer into one of the P2-GFP glomeruli, labeling the full complement of cells associated with that glomerulus (Fig. $1 B)$. After tracer injection, rhodamine-labeled neurons associated with the injected P2 glomerulus including ET cells and MT cells were clearly discernable (Fig. 1C). Through anterograde labeling, ET-cell axons were also clearly visible terminating beneath the P2-GFP glomerulus on the opposite side of the bulb (Fig. $1 B$ ). Antidromic stimulation of $\mathrm{P} 2$ intrabulbar projecting neurons was triggered using a small bipolar electrode inserted into the labeled terminal tufts (P2-IPLo) (Fig. $1 B, D$ ) and confirmed using whole-cell recordings at the ET-cell bodies (Fig. $1 E)$. 
A

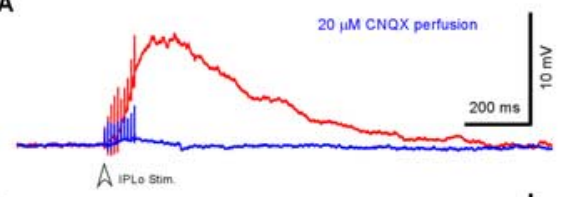

B

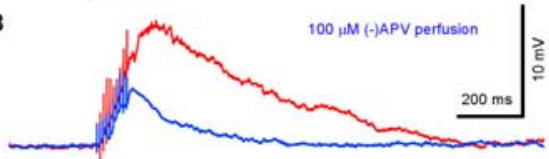

A IPLo stm

C

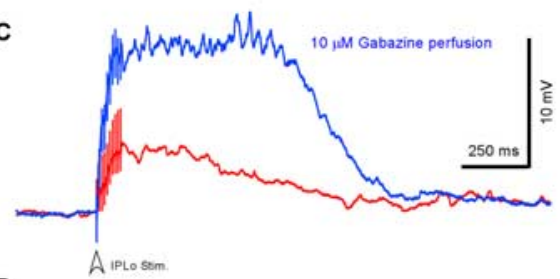

D

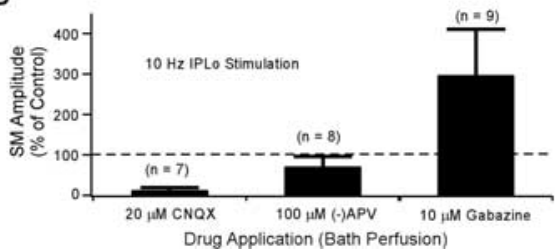

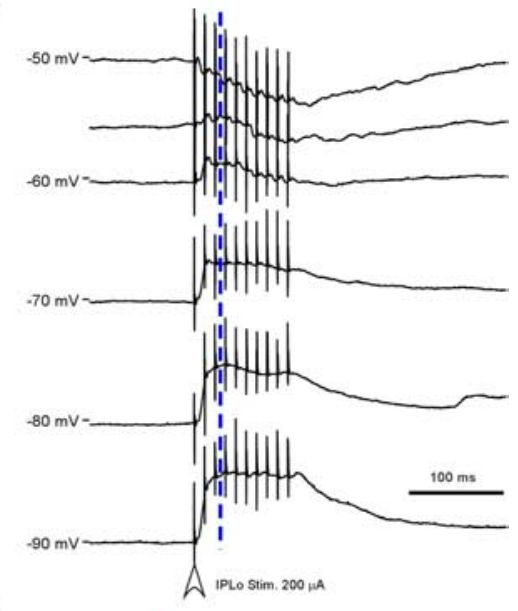

$\mathbf{F}$

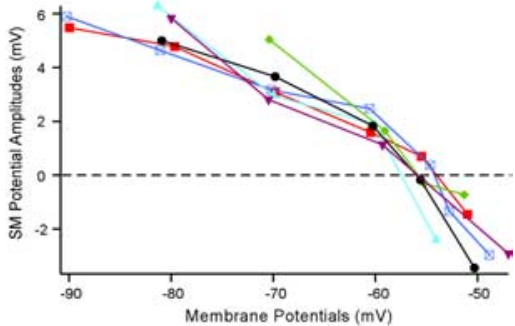

Figure 3. SM potentials are composed of both excitatory and inhibitory components and regulated by the mitral cell resting potential. $\boldsymbol{A}-\boldsymbol{C}$, SM potentials triggered by P2-IPLo stimulation $[0.2 \mathrm{~mA}, 0.5 \mathrm{~ms}$ at $100 \mathrm{~Hz}(\boldsymbol{A}, \boldsymbol{B}) ; 0.2 \mathrm{~mA}, 0.2 \mathrm{~ms}$ at $100 \mathrm{~Hz}(\boldsymbol{C})]$ were recorded from P2-MT cells using hemibulb slices, whereas neurotransmitter antagonists were applied to the bath. $\boldsymbol{A}$, Whole-cell recordings showed that application of the glutamate AMPA/kainate receptor antagonist, CNQX (20 $\mu \mathrm{M})$, virtually eliminated the SM response ( $12.1 \pm 6.8 \%$ of control, $n=7$; blue trace) compared with control [red trace; resting membrane potential (RMP), $-58 \mathrm{mV}$ ], suggesting that AMPA/kainate receptor signaling is necessary for initiation of the SM potential. $\boldsymbol{B}$, Perfusion of the NMDA receptor blocker $A P V(-)(100 \mu \mathrm{m})$ also produced a notable reduction in the SM response $(70.6 \pm 25.8 \%$ of control, $n=$ 8) compared with control (red trace; RMP, $-58 \mathrm{mV}$ ) but affected primarily the late depolarization phase of SM potential (blue trace). C, Conversely, application of gabazine $(10 \mu \mathrm{m})$, a $\mathrm{GABA}_{\mathrm{A}}$ receptor antagonist, produced an increase in the SM potential amplitude ( $296.6 \pm 114.1 \%$ of control, $n=9$, with $-40 \mathrm{pA}$ holding current; blue trace) compared with control (red trace; RMP, $-59 \mathrm{mV}$ ), revealing a large inhibitory component to the SM potential. $\boldsymbol{D}$, Graph quantifying the modulatory effects of the neurotransmitter blockers on the relative amplitude of the SM response showing that there is significant change in both the excitatory (CNQX, $p=0.0022 ; A P V, p=0.0259$ ) and inhibitory (gabazine, $p=0.000046$ ) components of the SM potential compared with control. In our perfusion system, the chemicals took full effects in $\sim 1 \mathrm{~min}$, and over $70 \%$ recovery was observed 8-10 min after chemical washout. $\boldsymbol{E}$, Whole-cell recording from a P2-MT cell held at various membrane potentials while eliciting IPLo-induced SM potentials show that the net-polarity is determined by the mitral cell resting potential. Traces reveal that the excitatory portion of the SM potential dominates the MT-cell response at hyperpolarized membrane potentials $(-60 \mathrm{mV}$ to -90 $\mathrm{mV})$, whereas the inhibitory component dominates the $S M$ response at more depolarized potentials $(-50 \mathrm{mV})$. Each trace is an average of three individual traces. $\boldsymbol{F}$, Graph showing the evoked SM potential amplitudes relative to the MT cells resting potential from six P2-MT cells, demonstrating a clear membrane voltage dependence in the polarity of SM response with an average measured reversal potential of $(-55.5 \mathrm{mV} \pm 1.3$; dotted line). Red squares represent data from the cell in $\boldsymbol{E}$, with measurements taken at the time point shown by the dotted line. Stim, Stimulation.

\section{Antidromic activation of ET cells produces a slow modulatory potential in MT cells}

To determine the role that intrabulbar projection neurons are playing in shaping glomerular output, we performed whole-cell recordings of mitral cells associated with the $\mathrm{P} 2$ glomerulus (P2-MT cells), while simultaneously exciting P2-ET cells at their IPLo site (Fig. $2 \mathrm{~A}$ ). We first observed that a single electrical pulse delivered to the IPLo beneath one P2 glomerulus produced a small EPSP in MT cells associated with the P2 glomerulus on the opposite side of the same bulb (Fig. $2 \mathrm{~B}$ ). Interestingly, a train of 10 pulses at $100 \mathrm{~Hz}$ produced a slow depolarizing potential that was similar to the EPSP observed with the single pulse but was greater in amplitude than the single pulse EPSP suggesting that this slow modulatory (SM) response may result from the summation of many smaller EPSPs (Fig. $2 B$ ).
To confirm the specificity of this SM response, we positioned the stimulating electrode in other regions of the bulb, including the IPLo beneath non-P2 glomeruli (Fig. 2A). Stimulation at no other site elicited an SM response in P2 mitral cells (Fig. 2D,E). To minimize nonspecific IPLo stimulation and avoid MT-cell excitation, we typically used $\sim 50 \%$ of the stimulus intensity required for maximum response. In many cases, single pulse stimulation at this low-level did not evoke a detectable EPSP at the MT cell body, whereas a train of low-level pulses produced an SM potential. Using a short train of 10 pulses (at $100 \mathrm{~Hz}$ ), we were able to elicit SM responses from MT cells in the majority of intact hemibulb preparations (61 of 106). Using a single IPLo pulse, the peak amplitude of the MT-cell EPSP was $3.9 \pm 2.2 \mathrm{mV}(n=19)$. The latency of the EPSP onset averaged $6.9 \pm 2.9 \mathrm{~ms}(n=19)$ and ranged from 3.1-13.5 ms, suggesting polysynaptic communication from ET to MT cells. By comparison, a train of 10 pulses at $100 \mathrm{~Hz}$ produced SM potentials of $5.1 \pm 2.7 \mathrm{mV}$ with the peak occurring at $124.6 \pm 102.7 \mathrm{~ms}$ and ranging from $6.5-$ $492.7 \mathrm{~ms}(n=61)$.

As the SM potential appeared to be an excitatory response, we next sought to determine the neurotransmitters and receptors that underlie its communication. We first tested the contribution of glutamate by applying glutamate receptor antagonists through bath perfusion. CNQX $(20 \mu \mathrm{M})$, an AMPA/kainate receptor antagonist, completely blocked all aspects of the SM potential (Fig. 3A), whereas the NMDA receptor blocker APV $(-)(100 \mu \mathrm{M})$ merely reduced the response rather than eliminated it (Fig. $3 B$ ). Interestingly, when we applied gabazine $(10 \mu \mathrm{M})$, a potent $\mathrm{GABA}_{\mathrm{A}}$ receptor antagonist, we observed a marked potentiation in the SM response, indicating that the SM potential also contains an inhibitory component (Fig. 3C). Together, these pharmacological data show that the SM potential is a compound signal composed of both excitatory and inhibitory components and that the AMPA/kainate glutamate receptors are critical to initiate the response.

\section{Mitral cell membrane potential determines the polarity of the SM potential}

The SM potential is composed of both excitatory and inhibitory components but is predominantly excitatory when evoked in MT cells at resting potential. Because the ion currents responsible for producing EPSPs and IPSPs are dependent upon electrochemical gradients within the cell, we hypothesized that the state of the MTcell membrane potential may determine whether the SM response is predominantly excitatory or inhibitory. To test this, we performed a set of experiments in which we induced SM potentials while varying the holding potential of the P2-MT cell. These results revealed clear 
voltage dependence in the net polarity of the SM response such that if the MT-cell membrane potential was held at a hyperpolarized state (i.e., -90 to $-60 \mathrm{mV}$ ), the $\mathrm{SM}$ response was predominantly excitatory (Fig. $3 E$ ). However, as the MT cell became more depolarized and was brought closer to its firing threshold (approximately $-45 \mathrm{mV}$ ) (Schoppa and Westbrook, 2002), the excitatory component decreased and the inhibitory component was revealed. By plotting the SM potential amplitude with respect to the membrane potential for multiple MT cells, we determined that the average reversal potential for the SM response in these cells was $-55.5 \pm 1.3 \mathrm{mV}(n=6)$, which is $4.4 \pm$ $1.6 \mathrm{mV}$ more depolarized than the resting potential of these MT cells (Fig. 3F). As MTcell resting potentials tend to vary (typically ranging between -55 and $-60 \mathrm{mV}$ ) so will the specific SM reversal potential point. Nevertheless, we propose that if the membrane potential of a given MT cell is below its SM reversal potential then eliciting an SM response in that cell would likely result in a depolarizing signal. However, if the MT-cell membrane potential were resting above its SM reversal potential, when an SM response was induced it would likely elicit a hyperpolarizing signal in the MT cell.

\section{The SM potential is mediated through the intraglomerular network}

Mitral cells have two distinct dendritic domains: a primary dendritic tuft that innervates the glomerulus and receives direct OSN input, and a set of secondary dendrites that extend laterally within the lower portion of the external plexiform layer and are thought to play an important role in modulating and tuning sensory input. To distinguish which of these dendritic domains are involved in mediating the SM potential, we used targeted pressure injections to apply CNQX directly into the P2 glomerulus. We found that blocking AMPA/kainate receptors at the primary dendrites was sufficient to completely eliminate the SM potential (Fig. 4A, C). Similarly, when we injected APV into the P2 glomerulus, we observed a reduction in the amplitude of the SM potential consistent with bath application of APV (Fig. 4B,C). Notably, APV had a significantly greater effect in reducing the train-evoked SM potential than the single stimulus-evoked potential ( $p=0.041$ ) (Fig. $4 D$ ), suggesting that the pulse train recruited more of the NMDA component than the single pulse. These experiments strongly suggest that the MT cell SM potential arising from ET-cell stimulation occurs primarily through intraglomerular communication and not through MT lateral dendrites. Furthermore, these data also confirm the results obtained by bath application showing that AMPA/kainate receptors are critical in producing the SM potential, whereas NMDA receptors play only a secondary role (Fig. $4 D$ ).

\section{Relative timing of the SM response determines whether it potentiates or inhibits $\mathrm{ON}$ excitation of mitral cells}

We have shown that the SM potential is transmitted through the glomerular network where it is ideally situated to influence the $0.0014, n=8)$. Stim, Stimulation.

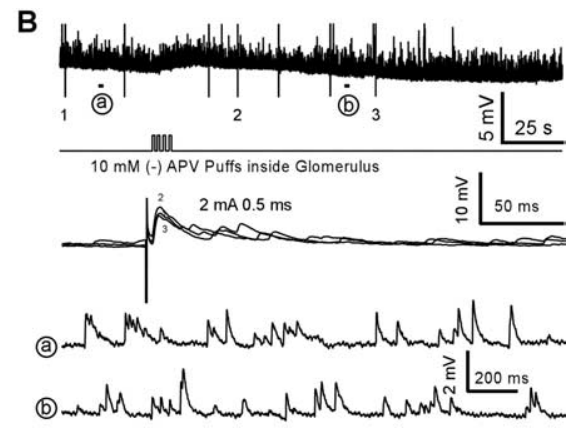

D

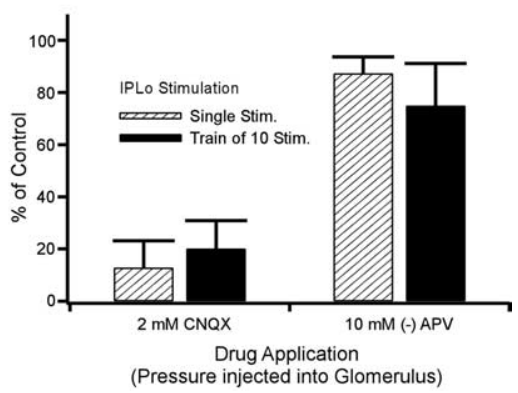

Figure 4. ET-cell-induced SM potential is communicated to MT cells through the glomerular network. Neurotransmitter antagonists were locally applied to hemibulb slices through pressure injection targeted directly into the P2 glomerulus, whereas P2-IPLo stimulation was used to evoke an SM potential. $\boldsymbol{A}$, Whole-cell recording from a P2-MT cell [resting membrane potential (

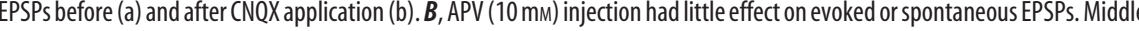
pottom response to a single stimulus (hatched bars; CNQX, $12.8 \pm 10.3 \%, p=0.00031, n=7 ; \mathrm{APV}, 87.3 \pm 6.4 \%, p=0.0012, n=7$ ) and to a train of stimuli (10 pulses at $100 \mathrm{~Hz}$, solid bars; CNQX, $20.1 \pm 10.8 \%, p=0.000013, n=13 ; \mathrm{APV}, 74.9 \pm 16.2 \%, p=$

initial MT-cell response to ON input. Because MT-cell action potentials are initiated at the axon hillock near the soma, it is reasonable to hypothesize that the SM potential, through its modulatory effects in the primary dendritic tuft, might also alter the firing frequency of MT cells in response to ON input. To test this hypothesis, we performed a series of dual activation experiments in which we stimulated both the ON and the IPLo terminating at the $\mathrm{P} 2$ glomerulus in different combinatorial sequences while performing whole-cell recordings from P2-MT cells. When IPLo stimulation preceded the ON stimulus, the resulting SM potential summated with the ON induced EPSP giving rise to a potentiated depolarizing response in the MT cell (Fig. 5B, top trace). Interestingly, when the IPLo stimulation followed the ON pulse, the EPSP evoked by ON stimulation was truncated, suppressing the depolarizing response in the MT cell (Fig. 5B, bottom trace). Thus, in either combinatorial sequence, the MT-cell response to the $\mathrm{ON}$ stimulus was significantly altered by the SM potential evoked by IPLo stimulation (Fig. $5 E$ ).

To test whether the SM potential could induce MT-cell firing in situations where sensory input alone would not evoke an action potential, we performed a set of experiments in which we adjusted the stimulus to the olfactory nerve such that it would produce a subthreshold EPSP in the P2-MT cell. When we preceded the ON stimulus with stimulation of the P2-IPLo site, we 

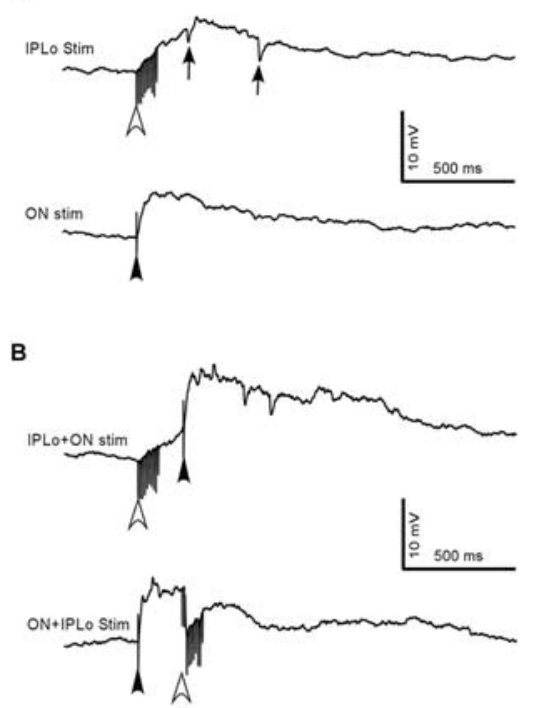

C

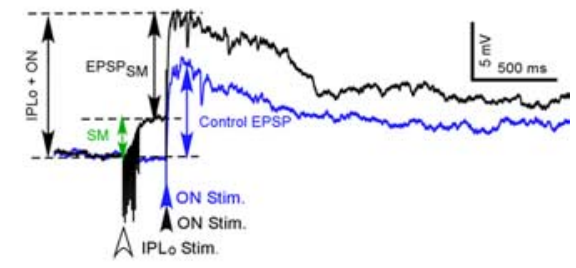

D

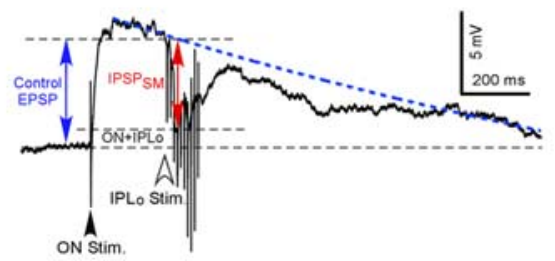

E

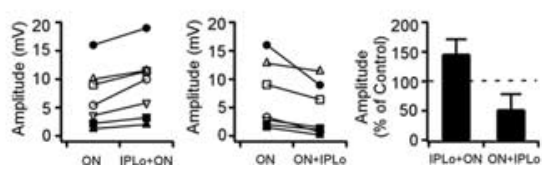

Figure 5. SM potential modulates olfactory nerve input to MT cell in a bidirectional manner. $\boldsymbol{A}$, Top trace, IPLo stimulusinduced SM potential $(0.5 \mathrm{~mA}, 0.5 \mathrm{~ms}, 100 \mathrm{~Hz}$, open arrowhead) producing a predominant EPSP signal with small IPSP elements riding upon the trace (arrows; resting membrane potential, $-54 \mathrm{mV}$ ). Bottom trace, Small EPSP response to a single stimulus (6 $\mu \mathrm{A}, 0.2 \mathrm{~ms}$, closed arrowhead) delivered to the $0 \mathrm{~N}$ leading to the P2 glomerulus. $\boldsymbol{B}$, Top trace, Sequential stimulation of IPLo (open arrowhead) and $\mathrm{ON}$ (closed arrowhead) using the same stimulus parameters applied above in $\boldsymbol{A}$ potentiates the mitral cell EPSP response. Bottom trace, Reversing the stimulus sequence such that IPLo stimulation (open arrowhead) follows an initial ON pulse (closed arrowhead) results in truncation of the EPSP signal. All traces in $\boldsymbol{A}$ and $\boldsymbol{B}$ are whole-cell recordings from the same P2-MT cell. C, Comparison of the P2-MT-cell EPSP response to a single ON pulse (blue trace) and the potentiated response resulting from the sequential stimulation of the P2-IPLo followed by an ON pulse (black trace). The potentiated amplitude (IPLO $+0 \mathrm{~N})$ is defined as the measured sum of the ON stimulus-induced EPSPS after the SM response (EPSP ${ }_{S M}$; black arrow) and the SM potential (SM, green arrow). D, Comparison of the P2-MT-EPSP response to a single ON pulse (blue trace) to the suppressed EPSP resulting from the reverse sequence stimulation of the $0 \mathrm{~N}$ followed by P2-IPLo (black trace). The suppressed maximum amplitude $\left(0 \mathrm{~N}+\right.$ IPLo) was measured by subtracting the IPSP $_{S M}$ (red arrow) from control EPSP (blue arrow). Dotted blue line, Fit curve of the EPSP decay with a single exponential equation. To more accurately represent a control decay procedure, we excluded the $800 \mathrm{~ms}$ of data points after the IPLo stimulus for this fitting. $\boldsymbol{E}$, Left and middle graphs show comparison amplitudes of ON-evoked EPSPs with sequential IPLo-stimulus-modulated EPSPs from seven individual MT cells. Left, IPLo stimulation induction before ON activation (IPLO + ON) increases membrane depolarization compared with ON activation alone. Middle, IPLo stimulation after ON activation suppresses the ON-evoked EPSPs. Summary histogram on right shows the percentage-change in the MT-cell EPSP response to the two sequential stimulus paradigms compared with $0 \mathrm{~N}$ stimulation alone. When SM responses preceded ON stimulation (IPLo $+0 N)$, the resulting EPSPs were significantly potentiated to $145.8 \pm 25.4 \%$ of control $\operatorname{EPSP}[p=0.032, n=7$; calculated by $($ IPLo $+0 \mathrm{~N}) /($ control EPSP) $\times 100 \%]$, although after an $0 \mathrm{~N}$ stimulus with an SM potential $(0 \mathrm{~N}+\mathrm{IPL} 0)$ suppressed resulting EPSPs to $51.5 \pm 26.5 \%$ compared with control EPSPs $[p=0.011, n=7$; calculated by $(0 \mathrm{~N}+\mid \mathrm{PL}$ L $) /($ control EPSP) $\times 100 \%]$. Stim, Stimulation.

reliably elicited bursts of action potentials from the MT cells $(n=$ 7) (Fig. 6A). These data suggest that activation of ET cells immediately before activation of MT cells could facilitate firing of the MT cells and thus enable transmission of sensory information centrally.

In a complementary set of experiments, we reversed the stimulus order by first delivering the $\mathrm{ON}$ stimulus and following it with P2-IPLo stimulation. In this configuration, the SM potential produced striking inhibition of the MT-cell response (Fig. $6 B$ ). By varying the delay of the IPLo stimulus with respect to the ON stimulus, we clearly revealed an inhibitory component of the SM potential (supplemental Fig. 1, available at www.jneurosci.org as supplemental material). Interestingly, this inhibitory component is capable of suppressing ON-induced MT-cell action potentials $(n=7)$ (Fig. 6B). Moreover, the SM potential is also capable of interrupting the depolarization-induced tonic firing of an MT cell by temporarily blocking action potentials when it is introduced $(n=5)$ (Fig. 6C). In combination, these data reveal that the coincident timing of olfactory stimuli with an SM potential is capable of either potentiating or inhibiting the transmission of olfactory information to the cortex.

\section{Spontaneously occurring SM-like potentials alter MT-cell activity}

Given that ET cells are bursting neurons and have been shown to fire action potentials spontaneously in a rhythmic manner (Hayar et al., 2004a), we recorded from MT cells to determine whether SM potentials occur without extrinsic stimulation. We performed whole-cell recordings and observed spontaneous slow depolarizing potentials that were remarkably similar both in amplitude and duration to IPLoevoked SM potentials within the same cells (Fig. 7A). Moreover, when spontaneous SM-like potentials occurred before a small depolarizing subthreshold electrical pulse (150 pA, $200 \mathrm{~ms}$ ) delivered to the MT-cell soma, the MT cell fired repetitive action potentials in response to the pulse (Fig. 7B). Similarly, when spontaneous SM-like potentials preceded small electrical pulses (20 $\mu \mathrm{A}, 0.5 \mathrm{~ms}$ ) delivered to the outer nerve layer, the combination easily evoked MT-cell action potentials (Fig. 7C). Finally, to confirm that MT cells associated with the same glomerulus not only receive the same sensory input (Schoppa and Westbrook, 2001) but also the same SM response, we performed dual whole-cell recordings from pairs of MT cells projecting to the P2 glomerulus. We show that both IPLo-evoked SM potentials and spontaneous SM-like potentials produce synchronously timed responses in the two MT cells that are similar to one another both in amplitude and duration (Fig. 7D). Thus, these data suggest that through SM potentials the spontaneous activity of ET cells can synchronously modulate the MT-cell output of individual glomeruli.

\section{Discussion}

Studying the local interactions between the various cell types in the olfactory bulb is often complicated by difficulties in identifying, selectively activating, and recording from individual neurons participating in the same local circuit. We have shown that through a unique hemibulb preparation we can selectively activate the ET cells associated with a particular set of glomeruli while simultaneously recording from the output neurons of the same glomeruli. Using this approach we, have uncovered that ET-cell activity elicits an SM potential within MT cells that occurs through a multisynaptic mechanism within the glomerular network. Our characterization of this SM potential has revealed that it is composed of both excitatory and inhibitory elements involving $\mathrm{AMPA} /$ kainate, $\mathrm{NMDA}$, and $\mathrm{GABA}_{\mathrm{A}}$ receptor signaling. Moreover, we demonstrate that through incident timing, with respect to sensory nerve activation, the SM potential is capable of either potentiating or suppressing the MT-cell response to olfactory nerve input. 
The SM potential is a polysynaptic response producing both excitation and inhibition of mitral cells

Although the precise connectivity by which the SM potential responds to both glutamate and GABA antagonists is unclear, it likely involves input from multiple juxtaglomerular cell types. This is consistent with our data showing that although a single IPLo stimulus is often insufficient to elicit an SM potential, a series of IPLo stimuli delivered sequentially can reliably produce an SM potential. In addition, the series of stimuli produce an SM potential that is typically larger than that of a single stimulus, possibly attributable to the recruitment and summation of multiple cells (Fig. 2). Although there is no clear evidence for direct synaptic connectivity between ET and MT cells, studies have shown a communication link between mitral/tufted cells through their primary dendrites (Urban and Sakmann, 2002). Thus, there are multiple sources within the glomerular domain capable of contributing to the excitatory component of the SM potential including ET cells, short axon cells, and OSNs. Interestingly, we demonstrate through application of CNQX that blocking glutamatergic transmission alone within the glomerular network results in loss of the excitatory without unmasking the inhibitory component of the SM potential (Fig. 4). This suggests that although a notable portion of this SM response is a GABA-mediated inhibitory signal, as revealed by gabazine application (Fig. 3C), activation of this inhibitory component is also dependent upon AMPA/kainate transmission. One explanation for this finding is that the inhibitory portion of the SM response is mediated by GABAergic PG cells, which synapse both with ET and MT cells (Shepherd et al., 2004), potentially bridging a communication gap between them. If so, then activation of these PG cells would rely upon glutamatergic signaling initiated by ET-cell activation. Thus, application of CNQX would not only block the excitatory portion of the SM potential but also indirectly block the PG-cellmediated inhibitory signaling to MT cells. It is also possible that other signaling mechanisms including synaptic spillover, gap junctions, and metabotropic receptors (i.e., metabotropic glutamate receptor, dopamine, GABAb) may also contribute to the SM response. Nevertheless, our pharmacological experiments clearly show that in MT cells, the SM potential is a net response to both excitatory and inhibitory synaptic inputs and requires AMPA receptor activation to initiate.

\section{Mitral cell membrane potential may mediate a "push/pull” mechanism}

Studies in both the visual and somatosensory systems examining the push/pull interaction between excitatory and inhibitory postsynaptic conductances have shown that the membrane potential of the output neuron can play a key role in determining its response (Priebe and Ferster, 2005; Wilent and Contreras, 2005). Similarly, we hypothesized that the depolarization state of the
B
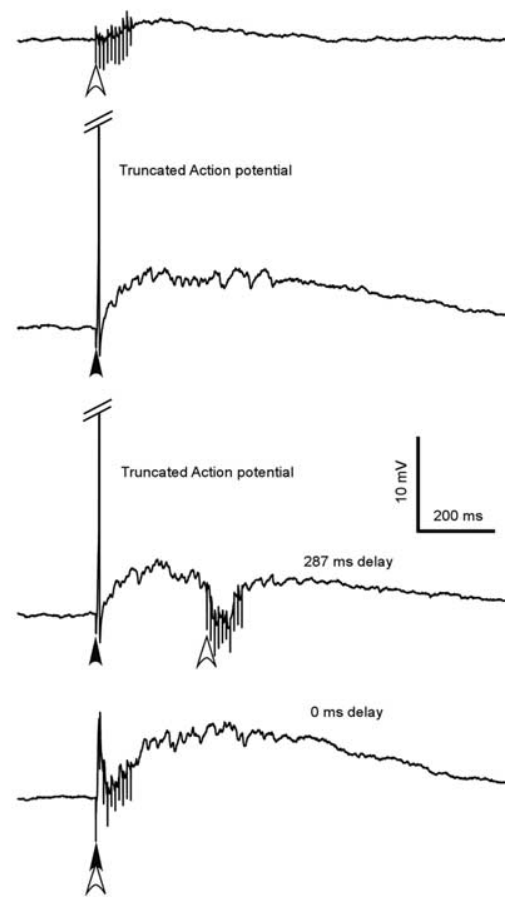

Figure 6. SM potential can either facilitate or suppress MT-cell firing. $A$, Whole-cell recording from a P2-MT cell reveals that although an IPLo-induced SM potential ( $1 \mathrm{~mA}, 0.5 \mathrm{~ms}$ at $100 \mathrm{~Hz}$; top trace) and a subthreshold $0 \mathrm{~N}$ stimulus ( $6 \mu \mathrm{A}, 0.2 \mathrm{~ms}$; middle trace) do not elicit mitral cell firing, sequential stimulation of IPL 0 and $0 N$ using the same stimulus intensities can elicit a burst of potential (second trace). When the ON and IPLo stimuli are delivered sequentially, the inhibitory component of the SM potential (n) IPLo-evoked SM response suppresses the tonic firings that occur when the P2-MT cell is depolarized by $140 \mathrm{pA}$ constant current (bottom trace; $n=5$ ). Sti, Stimulation.

MT cell itself may be important for regulating the SM response. We reasoned that if the SM potential is a compound synaptic signal that simultaneously integrates both EPSPs and IPSPs, then holding the MT cell at hyperpolarized membrane potentials (e.g., $-90 \mathrm{mV}$ ) should enhance the excitatory conductance while decreasing the inhibitory. Indeed, IPLo stimulation under these conditions elicited a predominantly excitatory SM response, most likely attributable to an increased glutamate component and a decreased GABA component. Conversely, at more depolarized potentials (e.g., $-50 \mathrm{mV}$ ) when MT cells are approaching their action potential threshold (approximately $-45 \mathrm{mV}$ ), the glutamate component decreases, whereas the GABA component increases such that IPLo stimulation produced a net inhibitory SM response. Thus, in Figure 3, we show that, much like the somatosensory cortical neurons, the membrane potential of the MT cells at the time of IPLo stimulation determines the net polarity of the SM response.

\section{ET-cell activity initiates SM potentials}

In our hemibulb preparation, we demonstrated that although the SM potential is a network response, evoked excitation of intrabulbar projecting ET cells alone is sufficient to trigger an SM response in MT cells. Interestingly, through whole-cell recordings, we show that MT cells experience spontaneous SM-like potentials when their primary dendritic tufts are maintained intact and connected to the glomerular circuitry (Fig. 7). We show that these SM-like potentials are physiologically and functionally equiva- 
A

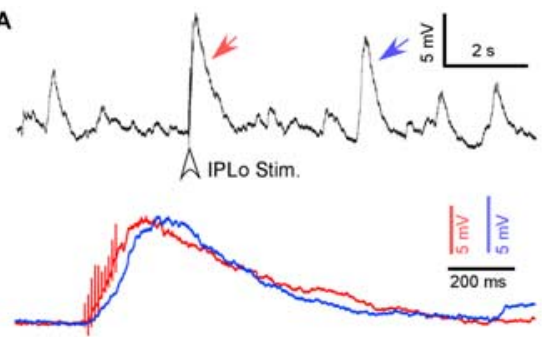

C

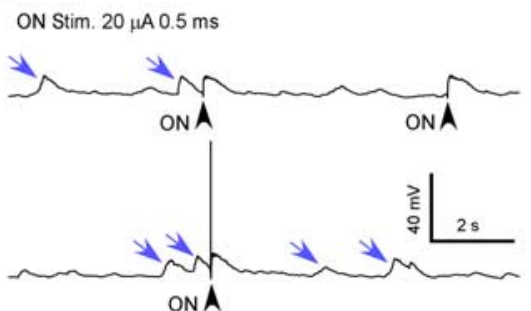

B
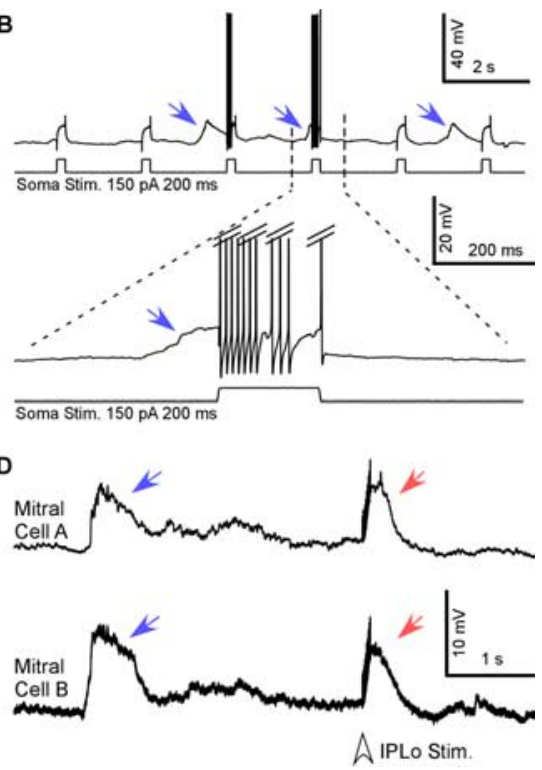

Figure 7. SM-like potentials capable of synchronizing MT-cell activity occur spontaneously. Whole-cell recordings from P2-MT cells using Hemibulb slice preparation reveal the $(\boldsymbol{A})$ presence of spontaneous SM-like potentials (one of which is indicated by the blue arrow) that occur without ON input and resemble IPLo stimuli-evoked SM potential (red arrow) both in amplitude and duration [resting membrane potential (RMP), $-55 \mathrm{mV}$; IPLo stimuli: $0.12 \mathrm{~mA}, 0.5 \mathrm{~ms}$ at $100 \mathrm{~Hz}$ ]. Recording trace segments are shown superimposed below (IPLo evoked, red; spontaneous, blue). $\boldsymbol{B}$, When spontaneous SM-like potentials (blue arrows) immediately preceded soma-induced membrane depolarization (150 pA, $200 \mathrm{~ms})$, the MT-cell response was amplified to produce action potentials (expanded region below; RMP, $-57 \mathrm{mV}$ ). C, Similarly, spontaneous SM potentials (blue arrows) immediately preceding subthreshold $0 \mathrm{~N}$ stimulus $(20 \mu \mathrm{A}, 0.5 \mathrm{~ms})$ also evoked MT-cell action potentials. $\boldsymbol{D}$, Dual whole-cell recordings from a pair of P2-MT cells that projected to the same glomerulus show a synchronous response to both IPLo stimulus-evoked SM potentials $(0.5 \mathrm{~mA}, 0.5 \mathrm{~ms}$, red arrow; RMP, $-59 \mathrm{mV})$ and spontaneous SM-like potentials suggesting that spontaneous SM-like potentials are being triggered by input of a common connection that may be used to coordinate MT activity. Similar results were observed in four other paired MT-cell recordings. Stim, Stimulation.

lent to evoked SM potentials. As in vitro studies have shown that ET cells are prone to spontaneous rhythmic-bursting activity (McQuiston and Katz, 2001; Hayar et al., 2004a; Antal et al., 2006; Zhou et al., 2006), it is likely that this activity is responsible for the spontaneous SM-like potentials. Thus, if spontaneous and evoked SM potentials are equivalent responses and ET cells behave similarly in vivo as they do in vitro then spontaneous ET-cell activity may also be an endogenous trigger for SM potentials in vivo.

\section{The SM potential may function to tune glomerular output}

In both somatosensory and visual cortex, studies have shown that the relative timing of excitatory and inhibitory synaptic input is used to tune neural response to establish stimulus selectivity (Priebe and Ferster, 2005; Wilent and Contreras, 2005). In our experiments, we show a similar dependence on timing such that the order in which multiple stimuli are received by an MT cell can determine its response. For example, if an SM response precedes an ON stimulus, the MT-cell response is potentiated, whereas if the SM response is presented either simultaneously or after an ON stimulus, the MT-cell signal is suppressed. This suggests that the SM potential alters MT-cell firing to tune the odor response of individual glomeruli. Because MT-cell-firing rate is linearly correlated to its membrane potential (Arevian et al., 2008) (supplemental Fig. 2, available at www.jneurosci.org as supplemental material), the SM potential is not only capable of determining MT-cell firing but could also be used to set the firing rate through small changes in membrane potential (Fig. 5). This would enable the SM potential to sharpen or enhance glomerular output, much

like the center-surround functions associated with retinal ganglion cells (Kuffler, 1953; Wässle and Boycott, 1991). In this case, the glomerulus would act as the center of an olfactory receptive field, albeit with less spatial constraint in the precise proximity of its inhibitory neighbors. Indeed, several studies have shown that the glomerular layer performs a contrast-enhancing function (Aungst et al., 2003; Vucinić et al., 2006), although the precise interplay of the connectivity has yet to be determined. As other studies have also described a centersurround mechanism within the deeper layers of the bulb (Yokoi et al., 1995; Luo and Katz, 2001; Galán et al., 2006; Schoppa, 2006), such a mechanism in the glomerular layer could potentially serve as a prefilter for deeper layer processing.

\section{Utilization of the SM response}

Although ET cells receive ON input that is similar to MT cells, we suggest that because ET cells have shorter primary dendrites, are more compact in size, and have much higher input resistance than mitral cells (Shepherd et al. 2004), it is likely that the timing and threshold of their response may differ from MT cells. In addition, as ET cells can exhibit long-lasting bursting activity (Hayar et al., 2004a), it is possible that the duration of the ET-cell response to odors also differs from that of MT cells. Our study has revealed an interesting timing-based modulatory mechanism in which ET-cell activation can determine MT-cell output. We hypothesize that the SM potential increases MT-cell response to weak odors through its potentiation ability while using its suppressive function during strong odor exposure to bring MT-cell membrane potentials back to resting level. This would facilitate the MT-cell response to subsequent breath cycles of an odor response and could be used to synchronize groups of MT cells responding to similar odorants. Thus, it will be interesting to see how such a mechanism functions in vivo.

\section{Concluding remarks}

Although OSN projections to the bulb are highly organized, giving rise to both molecular and functional maps, bulbar projections to the olfactory cortex are more spatially distributed (Wilson, 2001; Brunjes et al., 2005; Zou et al., 2005). As neural integration is also a feature of the olfactory cortex such that coincident MT-cell input is necessary to elicit firing of a given pyramidal neuron (Poo and Isaacson, 2007), it is likely that regulating MT-cell activity in response to an odorant is critical for enabling proper integration of olfactory signals. Such a mechanism could be partially mediated by an intraglomerular SM potential which enhances MT-cell activity that is temporally correlated to other components of the glomerular network while suppressing responses that are poorly correlated. We suggest that the SM potential plays an important role in this correlation process serving as a gate for MT-cell firing and thus working to synchronize glomerular output. 


\section{References}

Antal M, Eyre M, Finklea B, Nusser Z (2006) External tufted cells in the main olfactory bulb form two distinct subpopulations. Eur J Neurosci 24:1124-1136.

Arevian AC, Kapoor V, Urban NN (2008) Activity-dependent gating of lateral inhibition in the mouse olfactory bulb. Nat Neurosci 11:80-87.

Aungst JL, Heyward PM, Puche AC, Karnup SV, Hayar A, Szabo G, Shipley MT (2003) Centre-surround inhibition among olfactory bulb glomeruli. Nature 426:623-629.

Belluscio L, Lodovichi C, Feinstein P, Mombaerts P, Katz LC (2002) Odorant receptors instruct functional circuitry in the mouse olfactory bulb. Nature 419:296-300.

Brunjes PC, Illig KR, Meyer EA (2005) A field guide to the anterior olfactory nucleus (cortex). Brain Res Brain Res Rev 50:305-335.

Chen WR, Shepherd GM (2005) The olfactory glomerulus: a cortical module with specific functions. J Neurocytol 34:353-360.

Feinstein P, Mombaerts P (2004) A contextual model for axonal sorting into glomeruli in the mouse olfactory system. Cell 117:817-831.

Freeman WJ (1974) Stability characteristics of positive feedback in a neural population. IEEE Trans Biomed Eng 21:358-364.

Galán RF, Fourcaud-Trocmé N, Ermentrout GB, Urban NN (2006) Correlation-induced synchronization of oscillations in olfactory bulb neurons. J Neurosci 26:3646-3655.

Hayar A, Karnup S, Shipley MT, Ennis M (2004a) Olfactory bulb glomeruli: external tufted cells intrinsically burst at theta frequency and are entrained by patterned olfactory input. J Neurosci 24:1190-1199.

Hayar A, Karnup S, Ennis M, Shipley MT (2004b) External tufted cells: a major excitatory element that coordinates glomerular activity. J Neurosci 24:6676-6685

Hayar A, Shipley MT, Ennis M (2005) Olfactory bulb external tufted cells are synchronized by multiple intraglomerular mechanisms. J Neurosci 25:8197-8208.

Kuffler SW (1953) Discharge patterns and functional organization of mammalian retina. J Neurophysiol 16:37-68.

Lodovichi C, Belluscio L, Katz LC (2003) Functional topography of connections linking mirror-symmetric maps in the mouse olfactory bulb. Neuron 38:265-276.

Luo M, Katz LC (2001) Response correlation maps of neurons in the mammalian olfactory bulb. Neuron 32:1165-1179.

McQuiston AR, Katz LC (2001) Electrophysiology of interneurons in the glomerular layer of the rat olfactory bulb. J Neurophysiol 86:1899-1907.

Mombaerts P, Wang F, Dulac C, Chao SK, Nemes A, Mendelsohn M, Edmondson J, Axel R (1996) Visualizing an olfactory sensory map. Cell 87:675-686.

Poo C, Isaacson JS (2007) An early critical period for long-term plasticity and structural modification of sensory synapses in olfactory cortex. J Neurosci 27:7553-7558.

Priebe NJ, Ferster D (2005) Direction selectivity of excitation and inhibition in simple cells of the cat primary visual cortex. Neuron 45:133-145.

Ressler KJ, Sullivan SL, Buck LB (1994) Information coding in the olfactory system: evidence for a stereotyped and highly organized epitope map in the olfactory bulb. Cell 79:1245-1255.

Schoppa NE (2006) Synchronization of olfactory bulb mitral cells by precisely timed inhibitory inputs. Neuron 49:271-283.

Schoppa NE, Westbrook GL (2001) Glomerulus-specific synchronization of mitral cells in the olfactory bulb. Neuron 31:639-651.

Schoppa NE, Westbrook GL (2002) AMPA autoreceptors drive correlated spiking in olfactory bulb glomeruli. Nat Neurosci 5:1194-1202.

Shepherd GM (1963) Neuronal systems controlling mitral cell excitability. J Physiol 168:101-117.

Shepherd GM, Chen WR, Greer CA (2004) Olfactory bulb. In: The synaptic organization of the brain (Shepherd GM, ed). New York: Oxford UP.

Urban NN, Sakmann B (2002) Reciprocal intraglomerular excitation and intra- and interglomerular lateral inhibition between mouse olfactory bulb mitral cells. J Physiol 542:355-367.

Vassar R, Chao SK, Sitcheran R, Nuñez JM, Vosshall LB, Axel R (1994) Topographic organization of sensory projections to the olfactory bulb. Cell 79:981-991.

Vucinić D, Cohen LB, Kosmidis EK (2006) Interglomerular centersurround inhibition shapes odorant-evoked input to the mouse olfactory bulb in vivo. J Neurophysiol 95:1881-1887.

Wässle H, Boycott BB (1991) Functional architecture of the mammalian retina. Physiol Rev 71:447-480.

Wellis DP, Scott JW (1990) Intracellular responses of identified rat olfactory bulb interneurons to electrical and odor stimulation. J Neurophysiol 64:932-947.

Wilent WB, Contreras D (2005) Dynamics of excitation and inhibition underlying stimulus selectivity in rat somatosensory cortex. Nat Neurosci $8: 1364-1370$

Willhite DC, Nguyen KT, Masurkar AV, Greer CA, Shepherd GM, Chen WR (2006) Viral tracing identifies distributed columnar organization in the olfactory bulb. Proc Natl Acad Sci U S A 103:12592-12597.

Wilson DA (2001) Receptive fields in the rat piriform cortex. Chem Senses 26:577-584.

Yokoi M, Mori K, Nakanishi S (1995) Refinement of odor molecule tuning by dendrodendritic synaptic inhibition in the olfactory bulb. Proc Natl Acad Sci U S A 92:3371-3375.

Zhou Z, Xiong W, Masurkar AV, Chen WR, Shepherd GM (2006) Dendritic calcium plateau potentials modulate input-output properties of juxtaglomerular cells in the rat olfactory bulb. J Neurophysiol 96:2354-2363.

Zou Z, Li F, Buck LB (2005) Odor maps in the olfactory cortex. Proc Natl Acad Sci U S A 102:7724-7729. 